\title{
Derivation of the Relaxation Spectrum Representation of the Mechanical Response Function
}

\author{
Robert S. Marvin*
}

(April 3, 1962)

\begin{abstract}
Relaxation spectra have been used in both the presentation and interpretation of measurements of the mechanical properties of rubberlike polymers.
\end{abstract}

Relaxation spectra have been widely used in both the presentation and interpretation of measurements of the mechanical properties of rubberlike polymers. They are generally introduced in terms of a more or less specific model representing linear viscoelastic behavior, since the a priori assumption of a spectrum of a certain form is equivalent to assuming a certain model representation for mechanical response.

Ordinarily the derivation of such functions is based on the assumed equivalence of the mechanical response of a material to the response of an assembly of ideal springs and dashpots. And indeed it can be shown rigorously that any linear response is equivalent to the response of some such array if one admits certain limiting cases analogous to the familiar electrical cable with distributed resistance and capacitance. However, the proper form for the spectrum, whether it should be an integral or a sum, is not determined by this approach, but is arbitrarily assumed depending on the type of analysis preferred.

This ambiguity is not particularly serious for any practical application, since our experimental measurements are seldom precise or extensive enough to permit us to distinguish experimentally between the two forms. It is, however, quite possible to define the spectra unambiguously in terms of a relation between the transient and steady-state functions which must hold whenever the behavior is linear and the Boltzmann superposition principle applies. Moreover, this development automatically determines the nature of the spectrum in terms of the singularities of the steady-state response function, and yields directly an algebraic relation giving the spectrum exactly if the response function is known or assumed in analytic form.

The basic definition of the mechanical response function of a material is considered here to be that obtained from experimental observation. For a linear passive material either the response to a force which is a unit step function of time, $G(t)$, or the steady-state response to sinusoidal force, $G^{*}(i \omega) \equiv$ $G^{\prime}(\omega)+i G^{\prime \prime}(\omega)$, will completely define the response in shear. In principle $G(t), G^{\prime}(\omega)$, and $G^{\prime \prime}(\omega)$ can be measured for all real positive times, $t$, or frequencies, $\omega$, and we shall assume that both functions are known, are bounded, and are reasonably smooth.

\footnotetext{
*Present address: Department of Polymer Chemistry, Kyoto University,
} Kyoto. Japan.
Since we are considering relaxation-type behavior, we shall further assume that $G(t)$ is monotonic nonincreasing and contains no oscillatory terms which would characterize resonance- or damped resonance-type response.

We define a function $G^{*}(p)$, with $p=s+i \omega$, by analytic extension of $G^{*}(i \omega)$ from its line of definition. By a somewhat lengthy argument involving energy considerations it can be shown that the singularities of $G^{*}(p)$ are confined to the negative real axis including the origin. This same conclusion can be reached from a consideration of the relation between $G^{*}(p)$ and $G(t)$, established through the Carson transformation

$$
G^{*}(p)=p \int_{0}^{\infty} G(t) \exp (-p t) d t
$$

and its inverse

$$
G(t)=\frac{1}{2 \pi i} \int_{c-i \infty}^{c+i \infty}\left[G^{*}(p) / p\right] \exp (p t) d p .
$$

Since $G(t)$ is defined for all real $t \geq 0$, and has been assumed to have the character of a relaxation-type response, the singularities of $G^{*}(p) / p$ must be confined to the negative real axis, including the origin. Moreover, if these singularities are poles they must be of first order, since any higher order poles would lead to terms in $G(t)$ of the form $t^{b} e^{a t}$, inconsistent with relaxation-type behavior. If these singularities are simple poles, $G(t)$ will be given by a sum of terms of the form $r_{n} \exp \left(s_{n} t\right), s_{n} \leq 0$, where the sum may be either finite or infinite. This may be written in terms of a relaxation spectrum, $h(\lambda)$, as

if we take

$$
G(t)=\int_{0}^{\infty} h(\lambda) \exp (-\lambda t) d \lambda
$$

$$
h(\lambda)=\sum_{n} r_{n} \delta\left(\lambda+s_{n}\right) .
$$

The various molecular theories of viscoelastic behavior yield expressions of this form. However, a continuous spectrum is consistent with our assumptions, and will be found if $G^{*}(p)$ is characterized not by poles but by a branch cut along a portion or the whole of the negative real axis. Since the various approximation methods for obtaining a spectrum 
from the measured response functions ordinarily yield a continuous spectrum, it is of interest to see how this can be derived.

If $G^{*}(p)$ contains a branch cut, the contour of eq (1) may be deformed and the integral written as

$$
\begin{aligned}
G(t)= & (1 / 2 \pi i) \lim _{\rho \rightarrow 0} \lim _{\epsilon \rightarrow 0} \\
& \left\{\int_{-\infty}^{-\sqrt{\rho^{2}-\epsilon^{2}}}\left[G^{*}(s-i \epsilon) /(s-i \epsilon)\right] \exp [(s-i \epsilon) t] d s\right. \\
& +i \int_{-a}^{a} G^{*}\left(\rho e^{i \varphi}\right) \exp \left(\rho t e^{i \varphi}\right) d \varphi \\
+ & \left.\left.\int_{-\sqrt{\rho^{2}-\epsilon^{2}}}^{-\infty}\left[G^{*}(s+i \epsilon) /(s+i \epsilon)\right] \exp (s+i \epsilon) t\right] d s\right\}
\end{aligned}
$$

where $a=(\pi / 2)+\cos ^{-1}(\epsilon / \rho)$.

The second term of (4) yields $G^{*}(0)=G(\infty)$. The first and third terms can be combined, using the fact that $G^{*}$ of $p$-conjugate equals the conjugate of $G^{*}(p)$, to give:

$$
\begin{aligned}
G(t)-G(\infty) & =(1 / \pi) \int_{0}^{-\infty} d s\left(e^{s t} / s\right) \lim _{\epsilon \rightarrow 0} \operatorname{Im} G^{*}(s+i \epsilon) \\
& =\int_{0}^{\infty} d \lambda \exp (-\lambda t) h(\lambda)
\end{aligned}
$$

where

$$
h(\lambda)= \pm(1 / \pi \lambda) \lim _{\epsilon \rightarrow 0} \operatorname{Im} G^{*}(-\lambda \pm i \epsilon)
$$

This will be a continuous function of $\lambda$ over the region of the branch cut. If applied to a function characterized by simple poles it will yield zero for $h(\lambda)$ except at the poles where $\operatorname{Im} G^{*}(p)$ goes to infinity.

The same procedure can be used to define the retardation spectrum from the relation between $j(\infty)-j(t)$ and its transform, where $j(t)$ is the recoverable part of the creep function $J(t)$.

The algebraic relation (6) was derived by Gross ${ }^{1}$ by a less direct method starting from the integral giving $G^{*}(i \omega)$ in terms of $h(\lambda)$.

1 Bernhard Gross, Mathematical Structure of the Theories of Viscoelasticity. pp. 31-33 Hermann and Cie, Paris (1953). 